\title{
Sanguinarine Attenuates Neuropathic Pain by Inhibiting P38 MAPK Activated Neuroinflammation in Rat Model
}

This article was published in the following Dove Press journal: Drug Design, Development and Therapy

\author{
Chao $\mathrm{Yu}^{1,2, *}$ \\ Ping $\mathrm{Li}^{3, *}$ \\ Yan-Xiu Wang ${ }^{2}$ \\ Kai-Gang Zhang ${ }^{4}$ \\ Zun-Cheng Zheng ${ }^{3}$ \\ Li-Shuang Liang (iD) \\ 'Department of Pain Medicine, Qilu \\ Hospital, Cheeloo College of Medicine, \\ Shandong University, Jinan, Shandong, \\ People's Republic of China; ${ }^{2}$ Department \\ of Pain Medicine, Taian City Central \\ Hospital, Tai'an, Shandong, People's \\ Republic of China; ${ }^{3}$ Department of \\ Physical Medicine and Rehabilitation, \\ Taian City Central Hospital, Tai'an, \\ Shandong, People's Republic of China; \\ ${ }^{4}$ Department of Orthopaedic Surgery, \\ Taian City Central Hospital, Tai'an, \\ Shandong, People's Republic of China; \\ ${ }^{5}$ Department of Pain Medicine, Qilu \\ Hospital of Shandong University, Jinan, \\ Shandong, People's Republic of China \\ *These authors contributed equally to \\ this work
}

Correspondence: Li-Shuang Liang Department of Pain Medicine, Qilu Hospital of Shandong University, 44 Wenhua West Road, Jinan, Shandong Province 2500 I2, People's Republic of China

Tel/Fax +86 |356|764|3|

Email liangIs|224@I63.com

Kai-Gang Zhang

Department of Orthopaedic Surgery,

Taian City Central Hospital, No. 29 Long

Tan Road, Taian, Shandong 271000,

People's Republic of China

Tel/Fax +86 I5264832232

Email zhangkg0308@yeah.net
Background: Neuropathic pain seriously affects life quality, and it is urgent to develop novel drugs with high efficacy and few side effects. Sanguinarine (SG) is a natural plant medicine with anti-inflammatory and neuroprotection effects. This study aimed to investigate the effect of SG on chronic constriction injury (CCI)-induced neuropathic pain.

Materials and Methods: CCI rat model was established and rats were randomly divided into sham group, sham + SG group $(6.25 \mathrm{mg} / \mathrm{kg})$, CCI group, CCI + SG group $(1.00,2.50$ and $6.25 \mathrm{mg} / \mathrm{kg}$ ). The mechanical sensitivity and heat hypersensitivity of rats were monitored at different time points. Immunohistochemical, PCR, Western blot and ELISA were used to analyze p-p38 MAPK, NF- $\kappa$ B p65, TNF- $\alpha$, IL-1 $\beta$, and IL-6 levels.

Results: The mechanical sensitivity and heat hypersensitivity significantly reduced in rats of CCI group, but significantly increased in rats of CCI+SG group. TNF- $\alpha$, IL-1 $\beta$, and IL-6 levels significantly increased in the spinal cord of CCI rats, but significantly decreased in rats of $\mathrm{CCI}+\mathrm{SG}$ group. In addition, p38 MAPK activator antagonized beneficial effects of SG on neuropathic pain. Overexpression of $\mathrm{p} 38$ MAPK reduced the mechanical sensitivity and heat hypersensitivity, and enhanced NF- $\kappa$ B activity and the expression of inflammatory factors in CCI rats.

Conclusion: SG alleviates neuropathic pain via suppressing p38MAPK signaling and downregulating the expression of TNF- $\alpha$, IL-1 $\beta$, IL-6 and NF- $\kappa$ B activation. SG may be a potential therapeutic agent to treat neuropathic pain.

Keywords: sanguinarine, neuropathic pain, inflammatory cytokines, NF-kB, p38MAPK

\section{Introduction}

Neuropathic pain (NP) is characterized by hyperalgesia, spontaneous pain and allodynia, and is usually caused by physical sensory system damage or disease in peripheral or central nervous system. ${ }^{1}$ NP seriously affects life quality. Increasing lines of evidence have shown that p38 mitogen-activated protein kinase (MAPK) plays an active role in the occurrence and maintenance of hyperalgesia and hyperalgesia. ${ }^{2}$ Meanwhile, inflammatory factors play an important role in the occurrence and development of pain-related diseases. ${ }^{3}$ For example, interleukin- 6 (IL-6), tumor necrosis factor- $\alpha$ (TNF- $\alpha$ ), and interleukin-1 $\beta$ (IL-1 $\beta$ ) are involved in the occurrence and development of NP. ${ }^{4}$ In addition, p38 MAPK could mediate the activation of nuclear factor kappa $\mathrm{B}(\mathrm{NF}-\kappa \mathrm{B})$ and trigger inflammatory response. ${ }^{5}$

Sanguinarine (SG) (13-methyl $(1,3)$ benzodioxolo [5,6-c]-1,3-dioxolo [4,5-i] phenanthridinium) is an alkaloid extracted from the root of Sanguinaria canadensis 
and other poppy fumaria species. ${ }^{6,7}$ Recent evidence suggests that SG inhibits the occurrence and development of inflammation via the inhibition of the phosphorylation of p38 MAPK and the activation of NF- $\kappa$ B. ${ }^{8}$ However, there are few reports about the effect of SG on NP.

The aim of this study was to establish a rat model with chronic constriction injury (CCI) of the sciatic nerve to investigate whether SG exerts analgesic effects on NP, and to elucidate the underlying mechanism.

\section{Materials and Methods}

\section{Animals}

This study was carried out in strict accordance with the recommendations in the Guidelines for Shandong University. All experiments were approved by the Animal Ethics Committee of Shandong University following Chinese National Guidelines for Experimental Animal Welfare. Adult male Sprague Dawley rats (weight 180-200 g) were provided by Shandong Animal Experimental Animal Center and placed in a humidity control room at a temperature of $24 \pm 1{ }^{\circ} \mathrm{C}$ with light/ dark cycle for 12 hours. Animals had free access to food and water. All rats were randomly divided into six groups $(\mathrm{n}=8)$ : sham group, sham $+\mathrm{SG}$ group $(6.25 \mathrm{mg} / \mathrm{kg}), \mathrm{CCI}$ group, CCI + SG group (1.00, 2.50 and $6.25 \mathrm{mg} / \mathrm{kg})$. For CCI model, rats were anesthetized with isoflurane and placed in the lateral position and sciatic nerve was exposed, 4 sites were properly ligated with 3.0 chromic catgut, and the two ligation sites were separated by about $1 \mathrm{~mm}$. In sham group, sciatic nerve was exposed but not ligated. Immediately after surgery, sham + SG group $(6.25 \mathrm{mg} / \mathrm{kg})$ and CCI + SG group $(1.00,2.50$ and $6.25 \mathrm{mg} / \mathrm{kg})$ were intraperitoneally injected with SG $(1.00,2.50$ and $6.25 \mathrm{mg} / \mathrm{kg})$. Sham group and CCI group were injected with the same dose of $0.9 \%$ normal saline, once every two days.

For subgroup analysis, the rats were further divided into six groups $(\mathrm{n}=8)$ : sham group, intrathecal injection of $10 \mu \mathrm{L}$ normal saline daily for seven consecutive days; $\mathrm{CCI}$ group, intrathecal injection of $10 \mu \mathrm{L}$ normal saline daily for seven consecutive days; CCI + SB203580 group, intrathecal injection of $10 \mu \mathrm{L} 20 \mathrm{umol} / \mathrm{L}$ of SB203580 daily for seven consecutive days; CCI + N.S group, intrathecal injection of $10 \mu \mathrm{L}$ normal saline daily for seven consecutive days; CCI $+\mathrm{SG}(6.25 \mathrm{mg} / \mathrm{kg})$ group, intraperitoneal injection of SG $(6.25 \mathrm{mg} / \mathrm{kg})$ once every two days; CCI + SG $(6.25 \mathrm{mg} / \mathrm{kg})+$ anisomycin group, intraperitoneal injection of SG $(6.25 \mathrm{mg} / \mathrm{kg})$ once every two days and intrathecal injection of $10 \mu \mathrm{L} 20 \mu \mathrm{mol} / \mathrm{L}$ of anisomycin daily for seven consecutive days.

\section{Behavioral Assessment of Mechanical Sensitivity and Heat Hypersensitivity}

Based on the method of Vivancos et al, ${ }^{9}$ an electronic von Frey plantar aesthesiometer (Model: 2091, Yuyan Instruments Co., Ltd, Shanghai, China) was used to measure paw withdrawal mechanical threshold (PWT). Rats were placed on an elevated wire mesh, and the hind paw was vertically stimulated by the metal probe, and the stimulation intensity gradually increased, resulting in rapid paw contraction or paw licking within 3 seconds. The minimum stimulation intensity for the rat to produce a contraction response was recorded as PWT.

The heat hypersensitivity was measured using the plantar test. ${ }^{10}$ Rats were allowed to acclimate for 30 minutes before the test, then each rat was placed on an elevated glass platform and the heat source was located in the middle of the plantar of the left hind paw under the glass plate. The intensity of thermal stimulation was adjusted to reach $50{ }^{\circ} \mathrm{C}$ and the cut-off delay was set to $25 \mathrm{~s}$ in order to prevent tissue injury. The time from the start of stimulation to the time the hind paw is lifted, licked or avoided was recorded as thermal withdrawal latency (TWL). The behavioral tests were carried out before CCI surgery and 1, 3, 7 and 14 days after CCI.

\section{Immunohistochemical Staining}

The collected samples were fixed in 10\% neutral formaldehyde solution, embedded in paraffin, and cut into sections. After deparaffinization, gradient alcohol dehydration and antigen repair, the sections were blocked with normal goat serum, and then incubated with p-p38MAPK primary antibody (1:200, k20329-jhs, baileibo, China) overnight at $4^{\circ} \mathrm{C}$. After washing with PBS, the sections were incubated with to secondary antibody coupled to peroxidase enzyme and then stained with diaminobenzidine and hematoxylin. Three sections were randomly selected from each tissue sample. Each section was observed with $10 \times 40$ optical microscope in five fields. Image Proplus 6.0 image analysis system was used for analysis.

\section{Western Blot Analysis}

Spinal cord tissue was collected and then mechanically homogenized, Homogenates were centrifuged at $13,000 \mathrm{rpm}$ for $15 \mathrm{~min}$ at $4{ }^{\circ} \mathrm{C}$, and then the supernatant 
was collected. Protein concentration in the lysate was evaluated using Protein Assay Kit (PC0020, Solanum). Equal amounts of total protein were separated by electrophoresis on sodium dodecyl sulfate-polyacrylamide gel electrophoresis (SDS-PAGE) and transferred to polyvinylidene fluoride membrane (Merk Millipore, USA). The membrane was incubated with $5 \%$ skim milk for 2 hours at room temperature, then incubated with antibodies for p-p38 MAPK, NF- $\kappa$ B p65 and $\beta$-actin (all from Cell Signaling) at $4{ }^{\circ} \mathrm{C}$ overnight, and finally incubated with secondary antibodies. ChemiDoc MP imaging system (Bio-Rad) was used to detect protein expression, and IMAGE J software (version 1.47, USA) was used to analyze the gray levels.

\section{RT-PCR}

Total RNA was extracted from the spinal cord tissue using the Trizol (Quick-Start Protocol, Cat. Nos.74104), and treated with DNase I and isopropanol. Complementary DNA (cDNA) was synthesized with Oligo(dt) 12-18 using QuantiNovaTM Relieve Transcription kit (QuickStart Protocol, Cat. Nos.205411). PCR was performed using QuantiNova SYBR® Green PCR kit (Quick-Start Protocol, Cat. Nos.208054). The primers were as follows: NF- $\kappa$ B p65, 5'- GTGCAGAAAGAAGACATTG -3' (forward) and 5'- AGGCTAGGGTCAGCGTATGG -3' (relieve); $\beta$-actin, 5'-ACTCTGTGTGGATTG-GTGGC-3' (forward) and 5'-AGAAAGGGTGTAAAACGCAGC-3' (relieve). The results were analyzed by $2-\Delta \Delta \mathrm{Ct}$ method.

\section{Enzyme-Linked Immunosorbent Assay (ELISA)}

The dorsal horn of the spinal cord was collected and the contents of TNF- $\alpha$, IL- $1 \beta$ and IL- 6 were quantified by ratspecific ELISA kits (ELISA: MultiSciences, China) according to the manufacturer's instructions.

\section{Statistical Analysis}

All data were expressed as mean \pm standard error $(\mathrm{SD})$. The data were analyzed by ANOVA followed by post hoc test of LSD (if variance is equal) or Dunnett T3 (if variance is not equal). $P<0.05$ was considered statistically significant.

\section{Results}

\section{SG Improved PWT and TWL of CCI Rats}

Compared with the sham group (vehicle), the sham + SG group $(6.25 \mathrm{mg} / \mathrm{kg})$ showed no significant changes in PWT and TWL (Figure $1 \mathrm{~A}$ and $\mathrm{B}, \mathrm{n}=8, P>0.05$ ). As shown in
Figure $1 \mathrm{C}$ and D, compared with the sham group, PWT and TWL of CCI group significantly reduced after surgery $(n=8$, $* P<0.05$ ), but SG treatment significantly increased PWMT and PWTL in dose-dependent manner $(\mathrm{n}=8, P<0.05)$. These results indicate that the mechanical sensitivity and heat hypersensitivity significantly reduced in rats of CCI group, but significantly increased in rats of $\mathrm{CCI}+\mathrm{SG}$ group.

\section{SG Decreased Levels of Pro-Inflammatory Factors in Spinal Dorsal Horn of CCI Rats} ELISA showed that levels of IL-1 $\beta$ (Figure 2A), TNF-a (Figure 2B), and IL-6 (Figure 2C) in the dorsal horn of the spinal cord of CCI group significantly increased in CCI group compared with sham group $(P<0.05)$. However, intraperitoneal injection of SG $(1.00,2.50,6.25 \mathrm{mg} / \mathrm{kg})$ reduced the levels of TNF- $\alpha$, IL-1 $\beta$, and IL- 6 in a dose-dependent manner $(P<0.05)$. These results indicate that SG decreased levels of pro-inflammatory factors in spinal dorsal horn of CCI rats.

\section{SG Inhibited the Activation of P38 Protein in Spinal Dorsal Horn}

Immunohistochemical analysis showed that compared with Sham group, the activation of p38 MAPK (p-p38 MAPK staining) in spinal dorsal horn of rats in CCI group significantly increased. However, SG reduced p-p38 MAPK staining in $\mathrm{CCI}+\mathrm{SG}$ group $(1.00,2.50$ and $6.25 \mathrm{mg} / \mathrm{kg})$ in a dose-dependent manner (Figure $3 \mathrm{~A}$ and B).

Furthermore, Western blot analysis confirmed that p-p38 MAPK levels in spinal dorsal horn of rats in CCI group significantly increased, while SG significantly decreased p-p38 MAPK levels in CCI + SG group (1.00, 2.50 and $6.25 \mathrm{mg} / \mathrm{kg}$ ) in a dose-dependent manner (Figure 3C and D).

\section{SG Inhibited the Activation of NF- $\mathrm{KB}$ in Spinal Dorsal Horn}

RT-PCR and Western blot analysis showed that mRNA and protein levels of NF- $\kappa \mathrm{B}$ p65 in spinal dorsal horn of rats in CCI group increased significantly $(P<0.05)$. However, mRNA and protein levels of NF- $\kappa$ B p65 in the spinal dorsal horn of CCI rats decreased significantly after SG treatment in a dose-dependent manner $(P<0.05$, Figure 4A-C).

\section{SG Inhibited P38MAPK Activation to Downregulate NF- $\kappa B$ in $\mathrm{CCl}$ Rats}

To elucidate the mechanism by which $\mathrm{SG}$ inhibits the activation of NF- $\kappa$ B in CCI model, we focused on p38 MAPK pathway. Both SG and p38MAPK antagomir significantly 
A

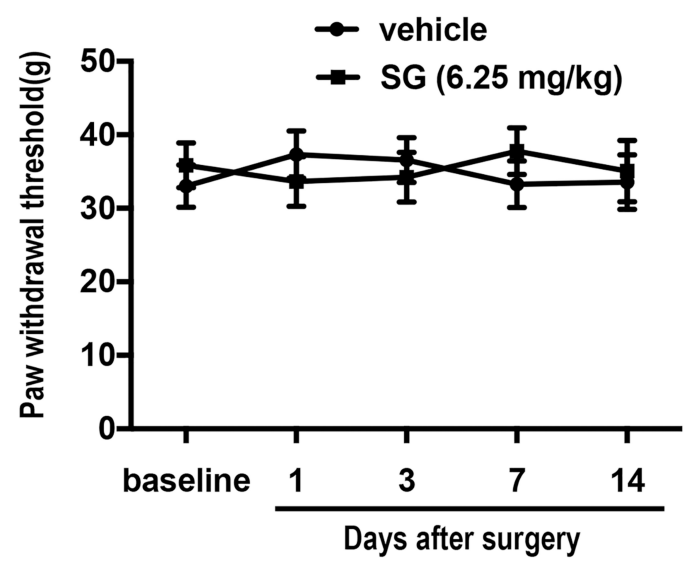

C

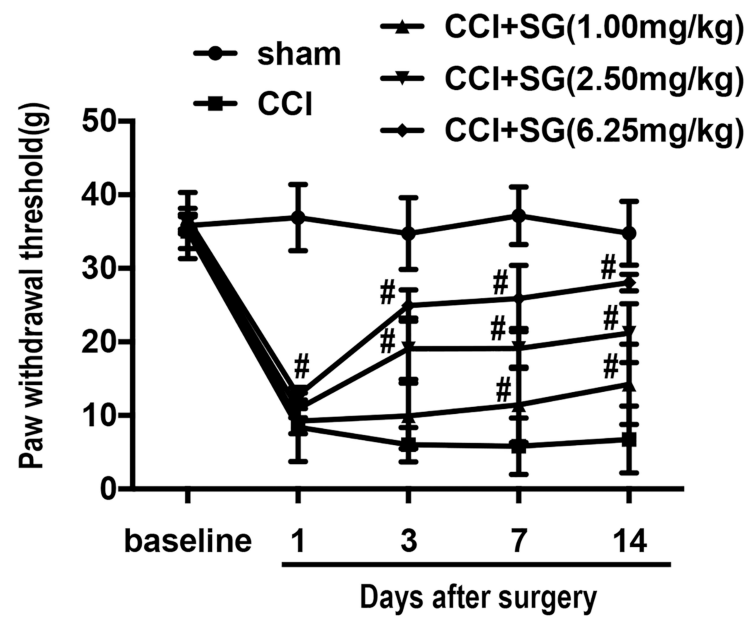

B

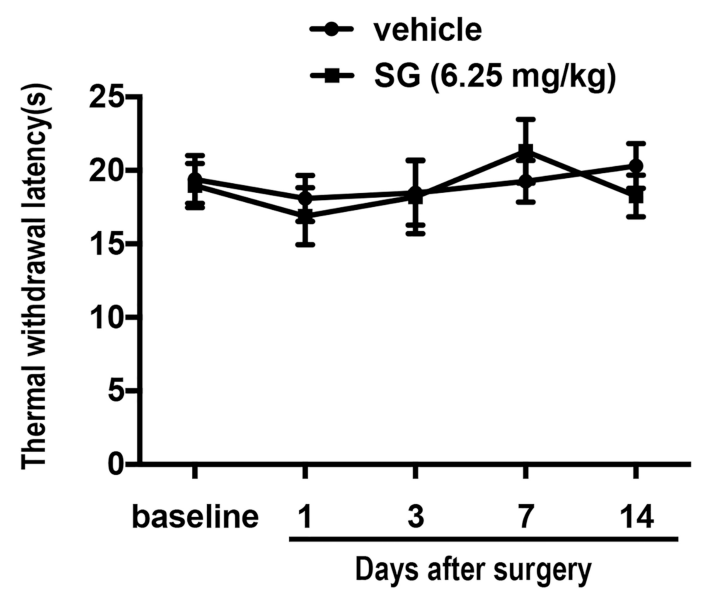

D

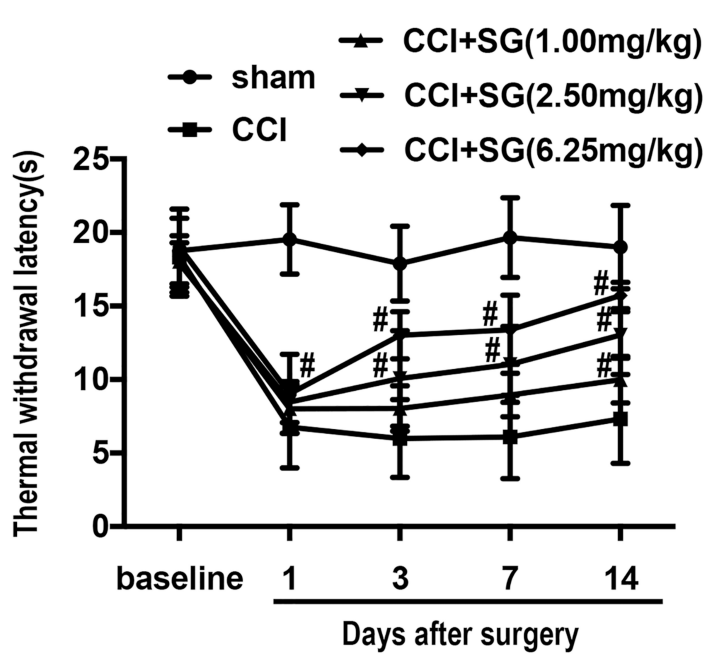

Figure I The effect of SG on PWT and TWL of CCI Rats. Compared with sham group, PWT and TWL did not change significantly after surgery $((\mathbf{A}$ and $\mathbf{B}), \mathrm{n}=8, \mathrm{P}>0.05)$. PWT (C) and TWL (D) in CCl group were significantly lower. PWT and TWL significantly increased in CCl $+\mathrm{SG}$ group $(1.00,2.50$ and $6.25 \mathrm{mg} / \mathrm{kg})$. ${ }^{\#}<<0.05 \mathrm{vs} \mathrm{CCl} \mathrm{group.}$ Abbreviations: SG, sanguinarine; CCl, chronic constriction injury; PWT, paw withdrawal threshold; TWL, thermal withdrawal latency.

A

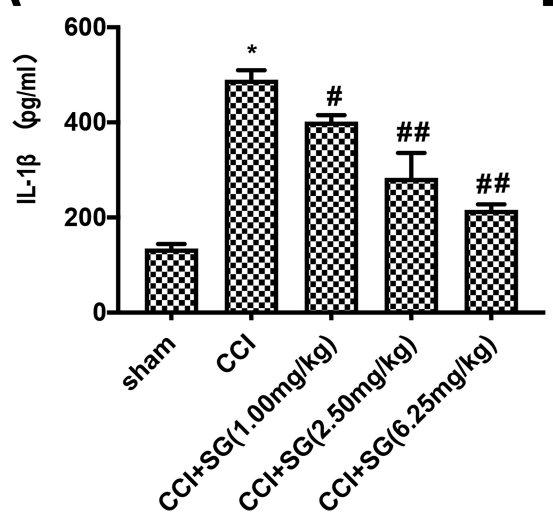

B

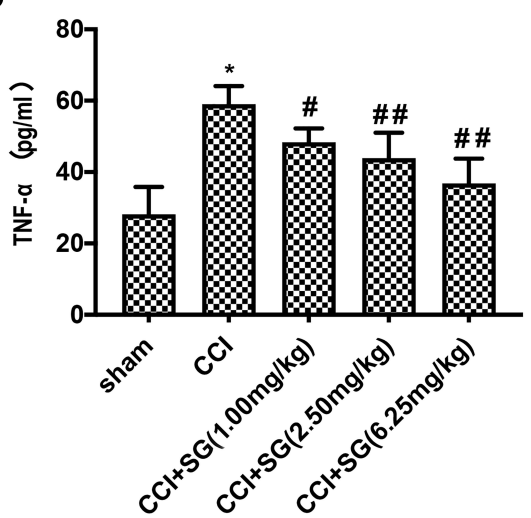

C

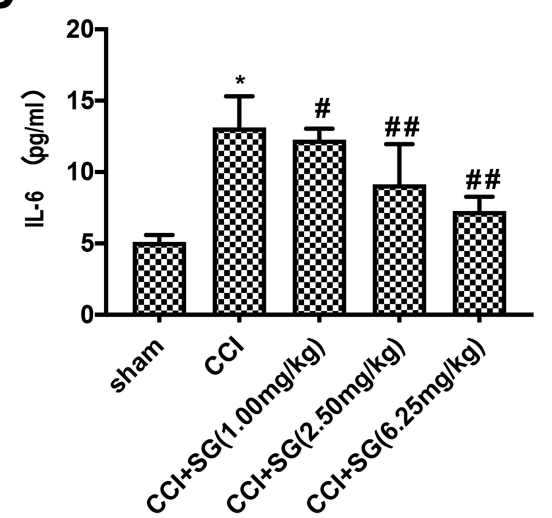

Figure 2 The effects of SG on IL-I $\beta$, TNF-a, and IL-6 levels in spinal dorsal horn of CCI Rats. The levels of IL-I $\beta$, TNF-a, and IL-6 levels in spinal dorsal horn were detected by ELISA. The levels of IL-I $\beta(\mathbf{A})$, TNF-a (B), and IL-6 (C) significantly increased in CCl group, but significantly decreased in CCI+SG group. Data are shown as mean \pm SD, $\mathrm{n}=3$. ${ }^{*} p<0.05$ vs sham group, ${ }^{\#} p<0.05$ and ${ }^{\# \#} p<0.01$ vs $\mathrm{CCl}$ group.

Abbreviations: SG, sanguinarine; $\mathrm{CCl}$, chronic constriction injury. 
A

sham

CCI

$\mathrm{CCl}+\mathrm{SG}(\mathrm{mg} / \mathrm{KG})$

1.00

2.50

6.25
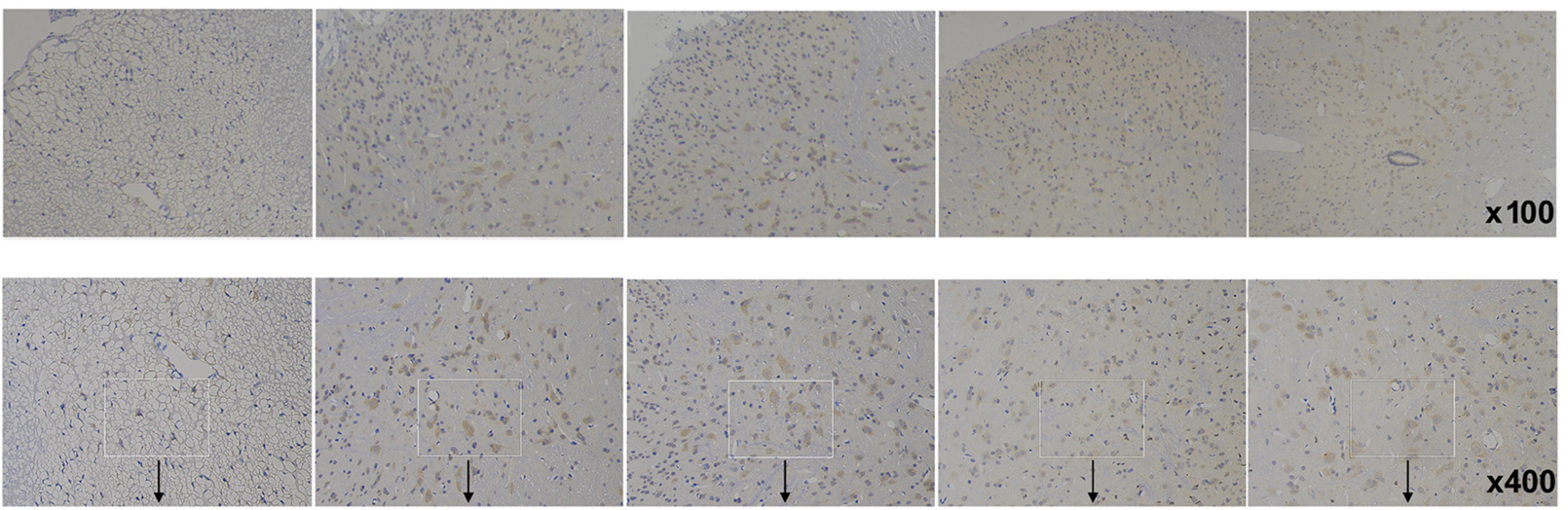

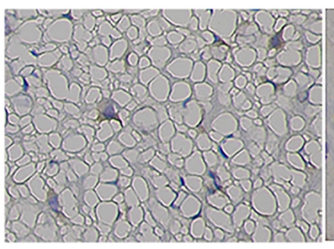

B

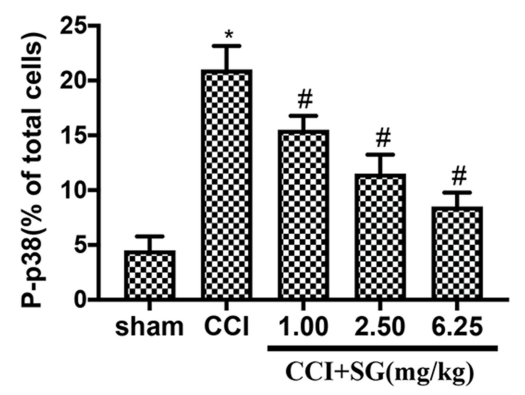

C

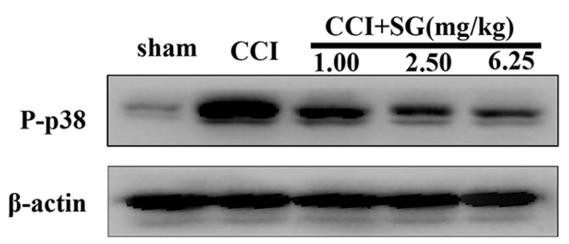

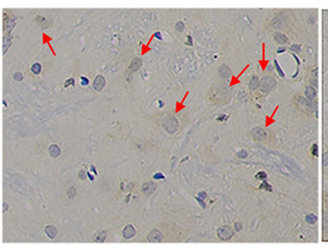

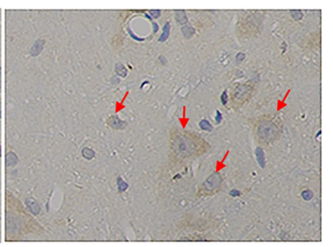

D

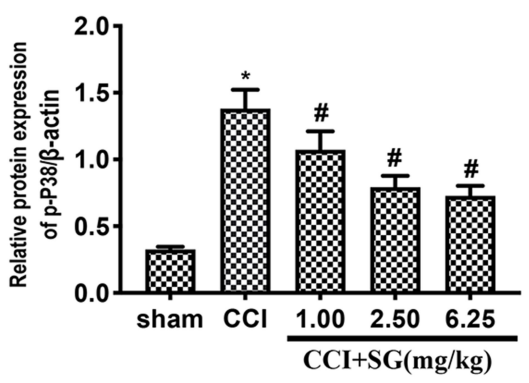

Figure 3 The effects of SG on the activation of p38 MAPK in spinal dorsal horn of CCI Rats. (A) Immunohistochemical staining of p-p38 protein in spinal dorsal horn. (B) Quantitative analysis of the percentage of positive p-p38 staining in all cells. (C) Western blot analysis of p-p38 protein levels in spinal dorsal horn. (D) Densitometry analysis of relative $p$-p38 level versus $\beta$-actin. Data are shown as mean $\pm S D, n=3$. Compared with the sham operation group $(* P<0.05)$, compared with $C C l$ group $\left({ }^{\#} P<0.05\right)$. Abbreviations: $\mathrm{SG}$, sanguinarine; $\mathrm{CCl}$, chronic constriction injury.

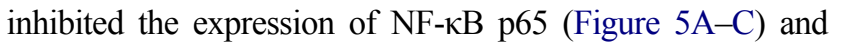
inflammatory factors (Figure 6A-C), while improved PWT and TWL significantly $(p<0.05)$ (Figure 6D-E). Consistently, p38MAPK agonists significantly increased the expression of NF- $\kappa$ B p65 (Figure 7A-C) and inflammatory factors (Figure $8 \mathrm{~A}-\mathrm{C}$ ), while reduced PWT and TWL $(p<0.05)$ (Figure 8DE). These results indicate that $\mathrm{SG}$ can inhibit neuroinflammation and the activation of NF- $\mathrm{KB}$ in $\mathrm{CCI}$ rats by inhibiting p38MAPK signaling.

\section{Discussion}

In this study, we chose CCI model to induce NP and found that PWT and TWL of CCI group were significantly lower than those of sham group. The reduced mechanical sensitivity and heat hypersensitivity in CCI model confirm successful establishment of rat model of NP. Based on this model we showed that SG treatment led to increased PWT and TWL in CCI rat model in a dose-dependent manner, indicating that SG can alleviate hyperalgesia in rats.

As we all know, inflammation is closely related to the occurrence and development of pain diseases, and proinflammatory factors play an important role in NP. ${ }^{11}$ In this study, the expression levels of IL-1 $\beta$, IL- 6 and TNF$\alpha$ were quantified by ELISA, and the results showed that IL-1 $\beta$, IL-6 and TNF- $\alpha$ levels in spinal cord tissue increased significantly in CCI model rat. However, after SG treatment, IL-1 $\beta$, IL-6 and TNF- $\alpha$ levels decreased 
A

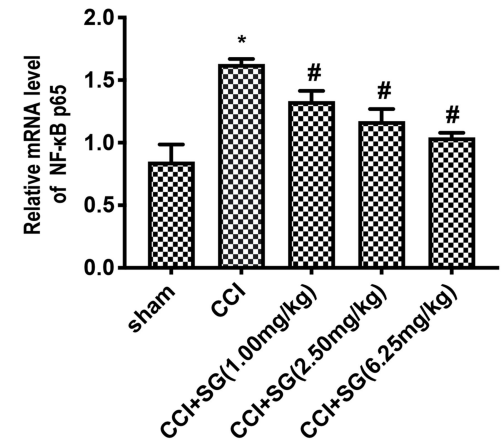

B

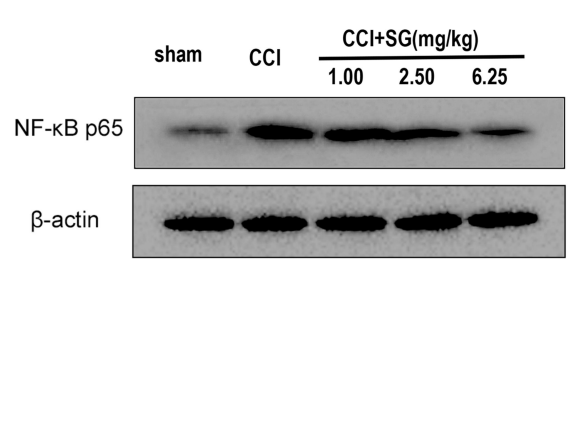

C

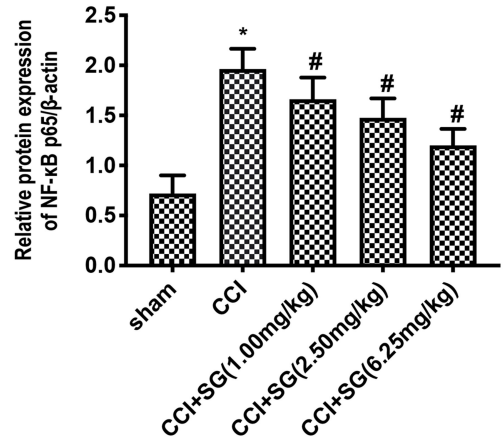

Figure 4 Effect of SG on NFкB p65 expression in spinal dorsal horn of CCl rats. (A) RT-PCR analysis of NF - $\kappa$ B p65 mRNA levels in the dorsal horn of the spinal cord. (B) Western blot analysis of NF - $\kappa$ B p 65 protein levels in the dorsal horn of the spinal cord. (C) Densitometry analysis of NF $-\kappa B$ p 65 protein levels in the dorsal horn of the spinal cord. Data are shown as mean $\pm S D, n=3$. Compared with the sham operation group ( $\left.{ }^{*} P<0.05\right)$, compared with $C C l$ group $\left({ }^{\#} P<0.05\right)$.

Abbreviations: SG, sanguinarine; $\mathrm{CCl}$, chronic constriction injury.

A

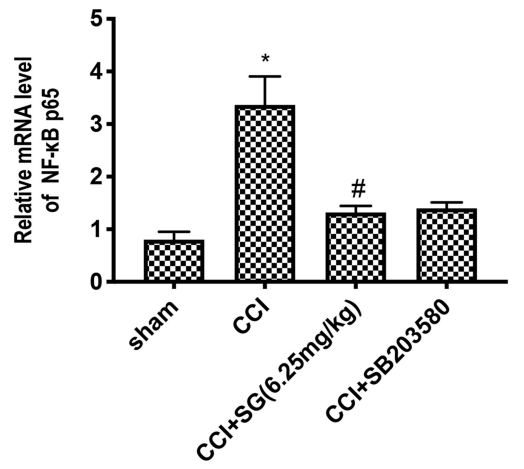

B

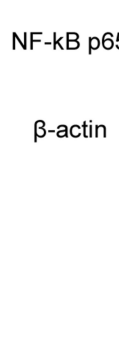

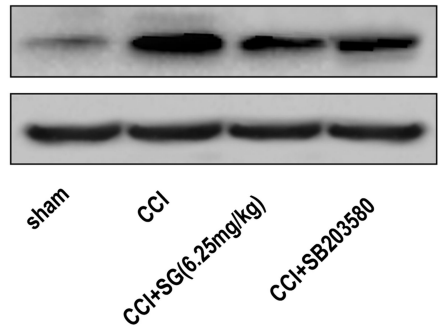

C

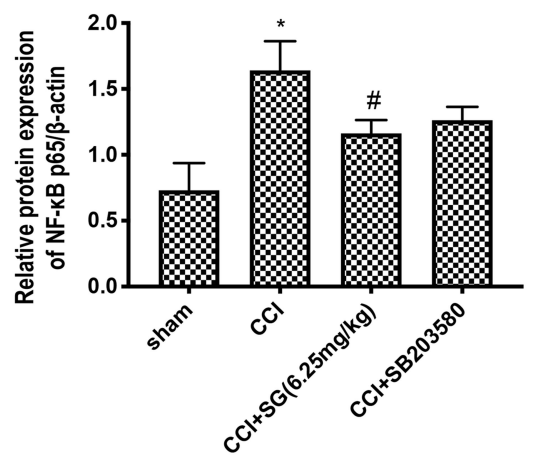

Figure 5 Effect of p38MAPK pathway inhibitor SB203580 on NF- $\kappa B$ in spinal cord of CCl rats. (A) RT-PCR analysis of NF - $\kappa$ B p65 mRNA levels in the dorsal horn of the spinal cord. (B) Western blot analysis of NF - $\kappa$ B p 65 protein levels in the dorsal horn of the spinal cord. (C) Densitometry analysis of NF - $\kappa$ B p65 protein levels in the dorsal horn of the spinal cord. Data are shown as mean $\pm S D, n=3$. Compared with the sham operation group $\left({ }^{*} P<0.05\right)$, compared with CCl group $\left({ }^{\#} P<0.05\right)$.

Abbreviations: $\mathrm{SG}$, sanguinarine; $\mathrm{CCl}$, chronic constriction injury.

significantly in CCI model rat. Therefore, these data indicate that $\mathrm{SG}$ could alleviate pain in neurogenic rat by reducing the expression of pro-inflammatory factors in spinal cord.

p38MAPK and NF- $\kappa$ B participate in the development and maintenance of NP. ${ }^{12}$ In one study, p38MAPK in spinal dorsal horn of CCI group was significantly activated compared to sham group. ${ }^{13}$ In another study on $\mathrm{CCI}$ rat model, NF- $\mathrm{KB}$ in spinal dorsal horn was upregulated to promote the expression and release of IL-1 $\beta$, IL-6, TNF- $\alpha$, resulting in NP. ${ }^{14}$ In this study, the results showed that compared with sham group, the expression levels of p38MAPK and NF- $\mathrm{B}$ p65 increased significantly in spinal dorsal horn of CCI group. However, the upregulation of p-p38 and NF- $\mathrm{kB}$ p65 in CCI model rat could be significantly inhibited by $\mathrm{SG}$ in a dosedependent manner.
The phosphorylation of $\mathrm{p} 38 \mathrm{MAPK}$ can upregulate proinflammatory factors IL-1 $\beta$, IL-6, TNF $\alpha \cdot{ }^{15-18}$ In the pathological circumstance, NF- $\mathrm{KB}$ can be translocated from the cytoplasm to the nucleus to induces the expression of pro-inflammatory factors in the downstream inflammatory pathway. ${ }^{14}$ In order to further verify whether SG alleviates neuropathic pain in CCI model rat through p38MAPK signaling, we treated model rat with p38MAPK activator and antagonist. The results showed that the activation of p38 MAPK could reduce PWT and TWL. Nevertheless, silencing p38 MAPK had the opposite effects and remarkably inhibited the activation of NF- $\mathrm{KB}$ and the expression of inflammatory factors TNF-a, IL-1B and IL-6, similar to the effects of SG treatment. Furthermore, overactivation of p38 MAPK attenuated the inhibitory effects of SG on the activation of NF- $\mathrm{KB}$ and the expression of inflammatory factors TNF-a, IL-1B and 
A

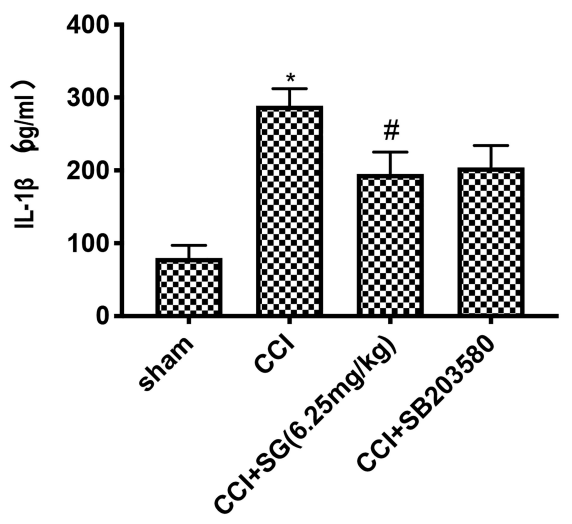

$\mathbf{E}$

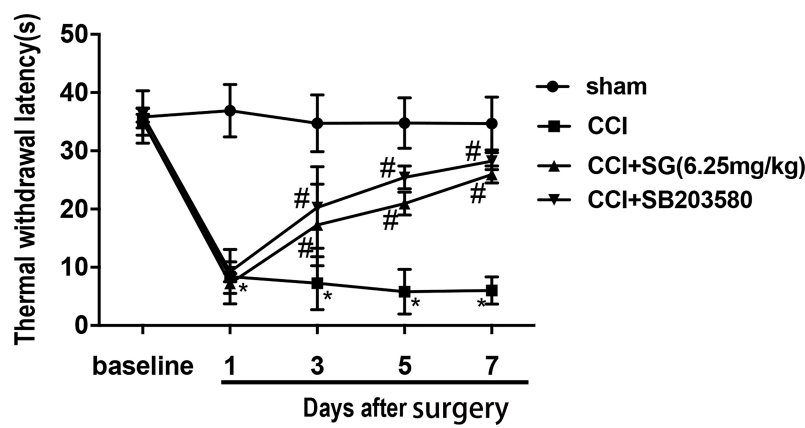

C

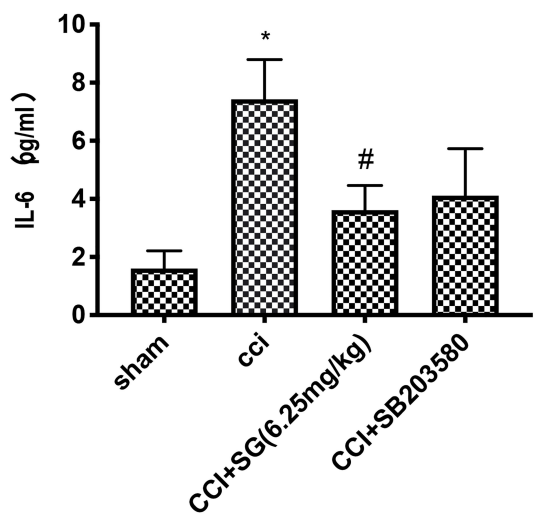

D

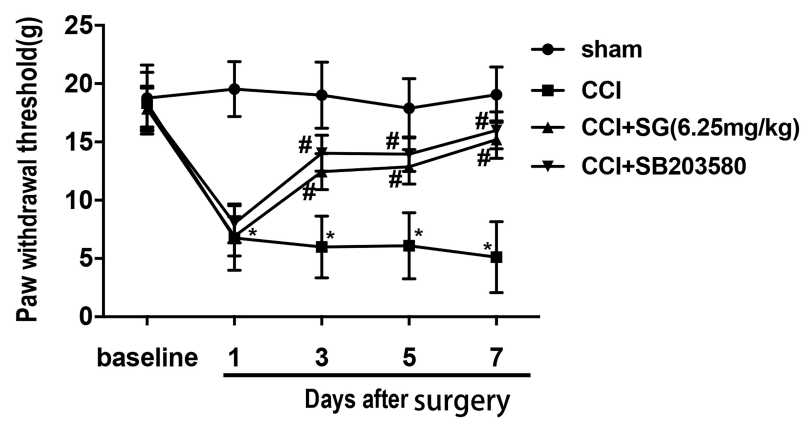

Figure 6 Effects of P38MAPK inhibitor SB203580 on inflammatory factors, PWT and TWL in CCI rats. (A-C). ELISA of inflammatory factors TNF-a, IL-IB and IL-6. The data are expressed as mean $\pm S D, n=3$. Compared with the sham group $(* P<0.05)$, compared with $C C l$ group $\left({ }^{\#} P<0.05\right)$. (D and $\left.E\right) P W T$ and TWL in each group. The data are expressed as mean $\pm S D, n=8$. Compared with the sham group $\left({ }^{*} P<0.05\right)$, compared with $C C l$ group $\left({ }^{\#} P<0.05\right)$.

Abbreviations: SG, sanguinarine; $\mathrm{CCl}$, chronic constriction injury; PWT, paw withdrawal threshold; TWL, thermal withdrawal latency.

A

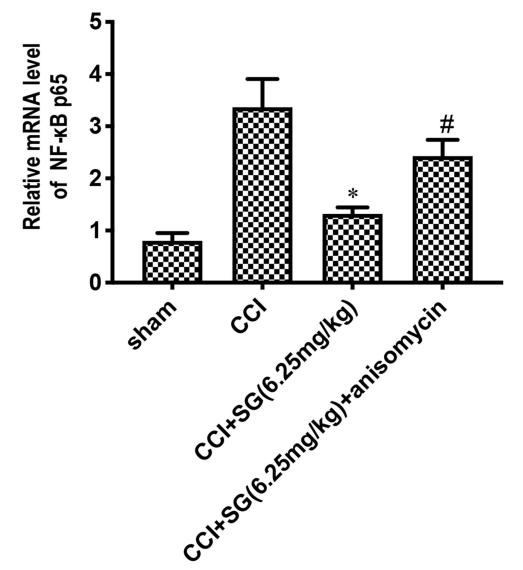

B

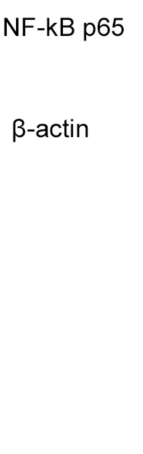

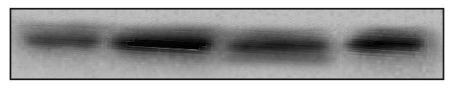

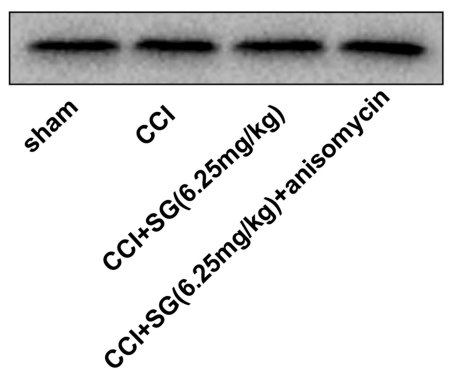

C

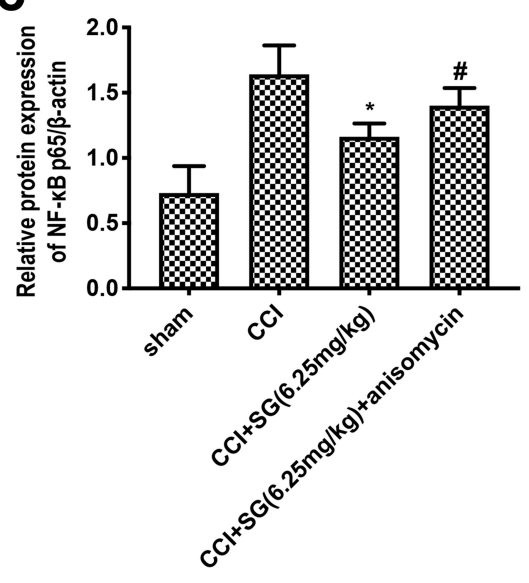

Figure 7 Effect of p38MAPK agonist anisomycin on NF- $\kappa$ B in spinal cord of CCl rats. (A) RT-PCR analysis of NF - $\kappa$ B p65 mRNA levels in spinal dorsal horn. (B) Western blot analysis of NF - $\kappa$ B p 65 protein levels in spinal dorsal horn. (C) Densitometry analysis of NF - $\kappa$ B p65 protein levels in spinal dorsal horn. Data are shown as mean \pm $\mathrm{SD}, \mathrm{n}=3$. Compared with sham operation group $\left({ }^{*} P<0.05\right)$, compared with $\mathrm{CCl}$ group $\left({ }^{\#} P<0.05\right)$.

Abbreviations: SG, sanguinarine; $\mathrm{CCl}$, chronic constriction injury.

IL-6. These results indicate that SG can exhibit antiinflammation effects through inhibiting p38 MAPK, consistent with previous findings. ${ }^{8}$
In summary, our study provides strong evidence that SG alleviates NP via suppressing p38MAPK signaling, NF- $\kappa \mathrm{B}$ activation and the expression of 

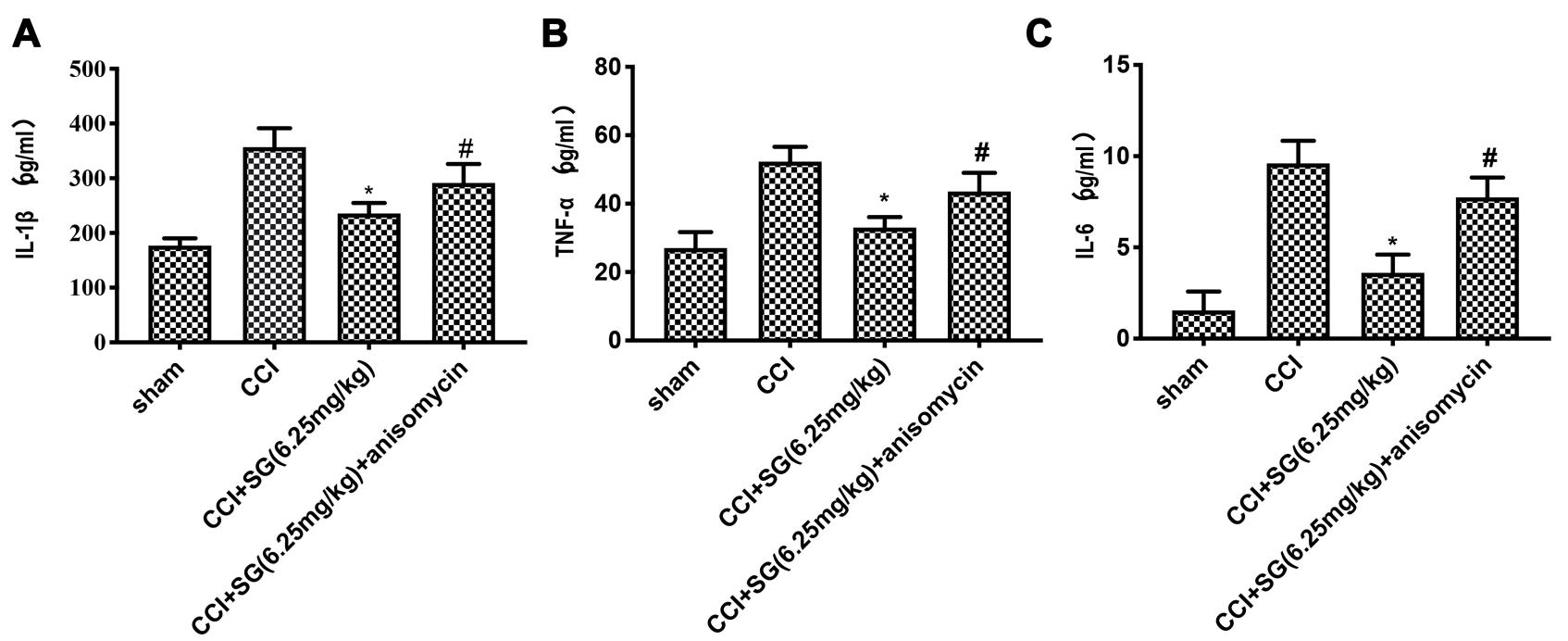

$\mathbf{E}$

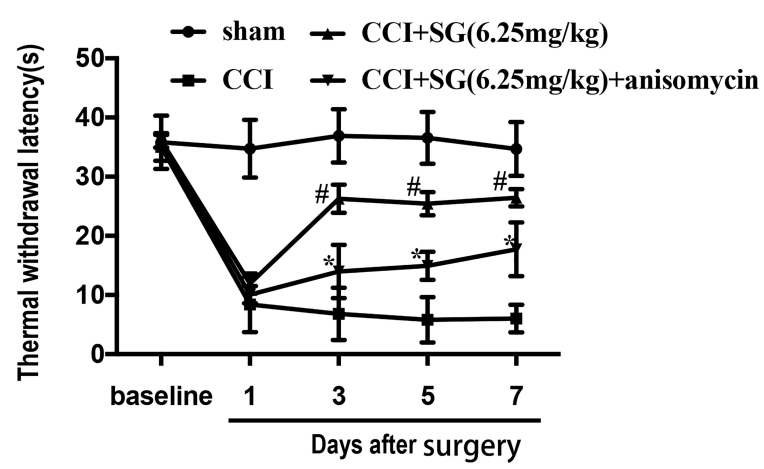

D

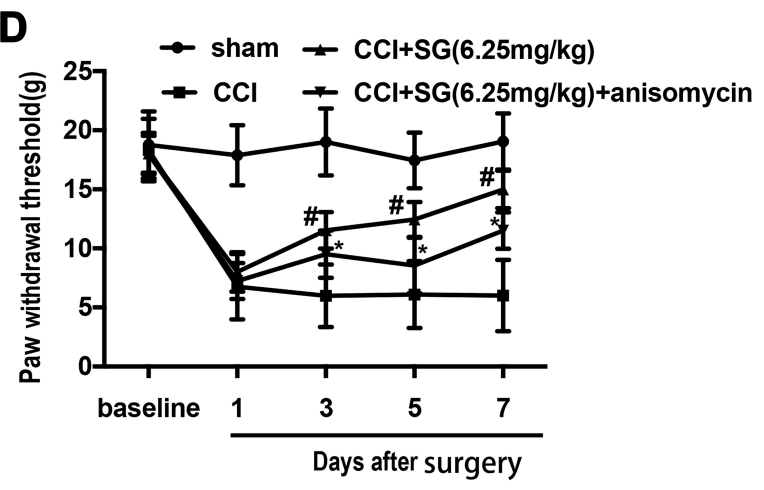

Figure 8 The effect of p38 MAPK pathway agonist anisomycin on inflammatory factors, PWT and TWL of CCI rats. (A-C). ELISA of inflammatory factors TNF-a, IL-IB and IL-6. The data are expressed as mean $\pm S D, n=3$. Compared with sham group ( $* P<0.05$ ), compared with $C C l$ group $\left.{ }^{\#} P<0.05\right)$. (D and $E$ ) PWT and TWL in each group. The data are expressed as mean $\pm \mathrm{SD}, \mathrm{n}=8$. Compared with $\mathrm{CCl}$ group, $* P<0.05$, compared with $\mathrm{CCl}+\mathrm{SG}(6.25 \mathrm{mg} / \mathrm{kg})$ group, ${ }^{\#} P<0.05$.

Abbreviations: SG, sanguinarine; $\mathrm{CCl}$, chronic constriction injury; PWT, paw withdrawal threshold; TWL, thermal withdrawal latency.

pro-inflammatory factors. Our new findings suggest that SG may be a potential therapeutic agent to treat NP.

\section{Acknowledgments}

The technical assistance of the Key Laboratory of Cerebral Microcirculation in Universities of Shandong (Taishan Medical University) is gratefully acknowledged.

\section{Funding}

This work was supported by the Natural Foundation of Shandong Province (no. ZR2017PH010) and Taian Science and Technology Guidance Project (no. 2017NS0143).

\section{Disclosure}

The authors have no conflicts of interest.

\section{References}

1. Jensen TS, Baron R, Haanpää M, et al. A new definition of neuropathic pain. Pain. 2011;152(10):2204-2205. doi:10.1016/j.pain.2011.06.017

2. Wang JY, Chen SP, Gao YH, Qiao LN, Zhang JL, Liu JL. Effect of repeated electroacupuncture intervention on hippocampal ERK and p38MAPK signaling in neuropathic pain rats. Evid Based Complement Alternat Med. 2015;2015:641286.

3. Munoz EM. Microglia-precursor cell interactions in health and in pathology. Biocell. 2018;42(2):41-45. doi:10.32604/biocell.2018.07 011

4. Old EA, Clark AK, Malcangio M. The role of glia in the spinal cord in neuropathic and inflammatory pain. Handb Exp Pharmacol. 2015;227:145-170.

5. Cai Y, Li W, Tu H, et al. Curcumolide reduces diabetic retinal vascular leukostasis and leakage partly via inhibition of the p38MAPK/NF- $\kappa$ B signaling. Bioorg Med Chem Lett. 2017;27(8):1835-1839. doi:10. 1016/j.bmcl.2017.02.045

6. Montes FQ, Vázquez-Hernández A, Fenton-Navarro B. Active compounds of medicinal plants, mechanism for antioxidant and beneficial effects. Phyton Int J Exp Bot. 2019;88:1-10.

7. Meng YY, Liu Y, Hu ZF, et al. Sanguinarine attenuates lipopolysaccharide-induced inflammation and apoptosis by inhibiting the TLR4/ NF-KB pathway in H9c2 cardiomyocytes. Curr Med Sci. 2018;38 (2):204-211. doi:10.1007/s11596-018-1867-4 
8. Deng W, Fang Y, Liu Y, et al. Sanguinarine protects against pressure overload-induced cardiac remodeling via inhibition of nuclear factorผB activation. Mol Med Rep. 2014;10(1):211-216. doi:10.3892/mmr 2014.2206

9. Vivancos GG, Verri Jr. WA, Cunha TM, et al. An electronic pressure-meter nociception paw test for rats. Braz J Med Biol Res. 2004;37(3):391-399. doi:10.1590/S0100-879X2004000300017

10. Hargreaves K, Dubner R, Brown F, Flores C, Joris J. A new and sensitive method for measuring thermal nociception in cutaneous hyperalgesia. Pain. 1988;32(1):77-88. doi:10.1016/0304-3959(88) 90026-7

11. Calvo M, Dawes JM, Bennett DL. The role of the immune system in the generation of neuropathic pain. Lancet Neurol. 2012;11 (7):629-642. doi:10.1016/S1474-4422(12)70134-5

12. Austin PJ, Moalem-Taylor G. The neuro-immune balance in neuropathic pain: involvement of inflammatory immune cells, immune-like glial cells and cytokines. J Neuroimmunol. 2010;229(1-2):26-50. doi:10.1016/j.jneuroim.2010.08.013

13. Cao J, Wang JS, Ren XH, Zang WD. Spinal sample showing p-JNK and P38 associated with the pain signaling transduction of glial cell in neuropathic pain. Spinal Cord. 2015;53(2):92-97. doi:10.1038/ sc. 2014.188
14. Sun T, Luo J, Jia M, Li H, Li K, Fu Z. Small interfering RNAmediated knockdown of NF-кBp65 attenuates neuropathic pain following peripheral nerve injury in rats. Eur J Pharmacol. 2012;682 (1-3):79-85. doi:10.1016/j.ejphar.2012.02.017

15. Fu ES, Zhang YP, Sagen J, et al. Transgenic inhibition of glial NF-kappa B reduces pain behavior and inflammation after peripheral nerve injury. Pain. 2010;148(3):509-518. doi:10.1016/j.pain.2010.01. 001

16. Saha RN, Jana M, Pahan K. MAPK p38 regulates transcriptional activity of NF-kappaB in primary human astrocytes via acetylation of p65. J Immunol. 2007;179(10):7101-7109. doi:10.4049/jimmunol. 179.10.7101

17. Hu Y, Liu Q, Zhang M, Yan Y, Yu H, Ge L. MicroRNA-362-3p attenuates motor deficit following spinal cord injury via targeting paired box gene 2. J Integr Neurosci. 2019;18(1):57-64.

18. Xu L, Huang Y, Yu X, Yue J, Yang N, Zuo P. The influence of p38 mitogen-activated protein kinase inhibitor on synthesis of inflammatory cytokine tumor necrosis factor alpha in spinal cord of rats with chronic constriction injury. Anesth Analg. 2007;105(6):1838-1844. doi:10.1213/01.ane.0000287660.29297.7b

\section{Publish your work in this journal}

Drug Design, Development and Therapy is an international, peerreviewed open-access journal that spans the spectrum of drug design and development through to clinical applications. Clinical outcomes, patient safety, and programs for the development and effective, safe, and sustained use of medicines are a feature of the journal, which has also been accepted for indexing on PubMed Central. The manuscript management system is completely online and includes a very quick and fair peer-review system, which is all easy to use. Visit http://www. dovepress.com/testimonials.php to read real quotes from published authors. 